\title{
Caracterização tecnológica de cookies produzidos com diferentes concentrações de farinha de algaroba durante armazenamento por 120 dias
}

\author{
Technological characterization of cookies made with different concentrations of \\ mesquite flour during 120 days of storage
}

\author{
Rennan Pereira de Gusmão1* (1), Thaisa Abrantes Souza Gusmão1, Henrique Valentim Moura1, \\ Maria Elita Martins Duarte', Mário Eduardo Rangel Moreira Cavalcanti-Mata' \\ ${ }^{1}$ Universidade Federal de Campina Grande (UFCG), Departamento de Engenharia de Alimentos, Campina Grande/PB - Brasil
}

\section{*Corresponding Author}

Rennan Pereira de Gusmão, Universidade Federal de Campina Grande (UFCG), Departamento de Engenharia de Alimentos, Rua Denise Alves de Medeiros, n60, CEP: 58410-743, Campina Grande/PB - Brasil, e-mail: rennangusmao@gmail.com

Cite as: Technological characterization of cookies made with different concentrations of mesquite flour during 120 days of storage. Braz. J. Food Technol., v. 21, e2017116, 2018.

Received: July 28, 2017; Approved: Oct. 05, 2017

\section{Resumo}

O objetivo deste trabalho foi avaliar os parâmetros de qualidade (firmeza, fraturabilidade, atividade de água e cor) de biscoitos enriquecidos com diferentes percentuais de farinha de algaroba (5\%, 15\% e 25\%), durante o armazenamento por 120 dias, além de determinar as características físicas (espessura, diâmetro, fator de expansão, volume específico e massa), físico-químicas (teor de água, cinzas, proteínas, açúcares, lipídios e fibras) e minerais (cálcio, fósforo e ferro). Os parâmetros de qualidade desses produtos foram acompanhados durante 120 dias de armazenamento, com intervalo de 15 dias entre as medições. Foram realizadas as caracterizações física e físico-química, e os minerais foram determinados por espectroscopia de difração de raios X por energia dispersiva. Os parâmetros de qualidade foram analisados estatisticamente, utilizando-se um delineamento experimental fatorial, com dois fatores: nove períodos de armazenamento e três formulações de biscoitos. Conclui-se que, após o período de 120 dias de armazenamento, houve diferença significativa entre os parâmetros de qualidade dos biscoitos. Os biscoitos elaborados com farinha de algaroba tiveram sua atividade de água, fraturabilidade e luminosidade aumentada, e sua firmeza e intensidade de amarelo e vermelho, reduzidas. Com o aumento da concentração de farinha de algaroba na formulação dos biscoitos, ocorreu uma variação maior entre a atividade de água inicial e a atividade de água final, e entre luminosidade inicial e luminosidade final. Os biscoitos enriquecidos com farinha de algaroba apresentaram nutrientes, como cálcio, ferro e fósforo, e características de qualidade aceitáveis durante o armazenamento, com comportamento similar a produtos já existentes no mercado.

Palavras-chave: Prosopis juliflora; Cookies; Qualidade; Armazenamento.

\section{Abstract}

This study aimed to assess the quality parameters (water activity, firmness, brittleness and colour) of cookies enriched with different mesquite flour concentrations $\left(5,15\right.$ and $\left.25 \mathrm{~g}^{100 \mathrm{~g}^{-1}}\right)$ during storage for 120 days, and to determine their physical (thickness, diameter, expansion factor, specific volume and mass), physicochemical (water, ash, protein, sugar, fat and fibre contents) and mineral (calcium, phosphorous and iron) characteristics. The quality parameters of these products were monitored for 120 days of storage, with an interval of 15 days between measurements. The physical and physicochemical characterizations were carried out and the minerals quantified using X-ray diffraction spectroscopy by dispersive energy. The quality parameters of the products were statistically analysed using a factorial experimental design with two factors, 9 stoarge periods and three cookie formulations. It was concluded there were significant differences between the quality parameters of the cookies after 120 days of storage. The water activity, brittleness and brightness increased in the cookies elaborated with mesquite flour and the firmness and intensities of yellowness and redness decreased. With the increase in 
Caracterização tecnológica de cookies produzidos com diferentes concentrações de farinha de algaroba durante armazenamento por 120 dias

Gusmão, R. P. et al.

concentration of mesquite flour in the cookie formulation, the difference between the initial and final water activities and between the initial and final brightness values increased. The cookies enriched with mesquite flour showed high concentration of some nutrients (calcium, iron and phosphorus) and acceptable quality characteristics during storage, with similar behavior to products already existing on the market.

Keywords: Prosopis juliflora; Cookies; Quality; Storage.

\section{Introdução}

Na literatura, é possível encontrar diversos trabalhos que estudaram a substituição parcial ou total do trigo em produtos de panificação (MARTINEZ et al., 2014; LEMOS et al., 2012; RAMOS et al., 2012), seja para melhoria da qualidade nutricional, seja para atender a um público específico de portadores de doenças nutricionais, como os celíacos, e a um público que cada vez mais busca produtos com atrativos mais saudáveis, como a presença de fibras e compostos bioativos.

Segundo Robertson (2006), muitas são as variações de qualidade dos alimentos industrializados, durante o seu período de armazenamento. Mesmo tendo a deterioração microbiana sob controle, outras reações, como alterações de cor, sabor, textura, qualidade nutricional e funcional, podem comprometer o alimento. Os fatores que influenciam a velocidade das reações de transformação são vários: temperatura, teor de água, atividade de água, acidez, teor de oxigênio, estado da matriz sólida, presença de catalisadores, entre outros.

Dependendo das condições de armazenamento, o biscoito pode sofrer alteração, devido à adsorção de água, que acarretará uma mobilidade maior das moléculas, passando do estado vítreo para o gomoso (transição vítrea), caracterizado por uma elevada mobilidade molecular. Neste estado, ocorrem alterações na qualidade do produto, como a perda da crocância característica, além de este se tornar um meio mais propício para o crescimento microbiano.

Diante do exposto, este trabalho teve, como objetivo, avaliar os parâmetros de qualidade (atividade de água, firmeza, fraturabilidade e cor) de biscoitos formulados com diferentes percentuais de farinha de algaroba (5, 15 e 25\%), durante o armazenamento por 120 dias, além de determinar suas características físicas (espessura, diâmetro, fator de expansão, volume específico e massa), físico-químicas (teor de água, cinzas, proteínas, lipídios e fibras) e minerais (cálcio, fósforo e ferro).

\section{Material e métodos}

\subsection{Local dos experimentos}

Os experimentos foram realizados nos Laboratórios de Engenharia de Alimentos da Universidade Federal de Campina Grande e no Laboratório de Panificação SENAI-PB.

\subsection{Recepção e moagem da algaroba}

As amostras de algaroba madura [Prosopis juliflora (Sw.) DC.], obtidas na cidade de Serra Branca, Paraíba, foram recebidas e higienizadas com água clorada a 30 ppm. Em seguida, a algaroba foi submetida ao processo de secagem convectiva, utilizando temperatura de $60{ }^{\circ} \mathrm{C}$, durante oito horas. Após a secagem, o produto foi submetido à operação unitária de moagem, em processo de batelada (10 g), no moinho de facas, fabricante Tecnal, com seu conjunto de facas regulado para $1 \mathrm{~mm}$, utilizando-se peneira de 10 Mesh. Após a moagem, a farinha de algaroba, com teor de água de 7\% (base úmida) foi acondicionada em embalagem hermética de polietileno, mantida em temperatura ambiente de $25^{\circ} \mathrm{C} \pm 3,0^{\circ} \mathrm{C}$.

\subsection{Formulação dos biscoitos}

Foram desenvolvidas três formulações de biscoitos, encontradas na Tabela 1 (experimentos 1, 2 e 3), utilizando-se farinha de algaroba com teor de água ( $7 \%$ b.u). Os ingredientes utilizados para elaboração dos biscoitos foram: farinha de trigo sem fermento (M. DIAS BRANCO S.A, Fortaleza-CE),

Tabela 1. Formulação para produção dos biscoitos.

\begin{tabular}{|c|c|c|c|}
\hline Ingrediente & $\begin{array}{c}\text { Experimento } 1 \\
\left(\mathrm{~g}^{\left.100 \mathrm{~g}^{-1}\right)}\right.\end{array}$ & $\begin{array}{c}\text { Experimento } 2 \\
\left(\mathrm{~g} .100 \mathrm{~g}^{-1}\right)\end{array}$ & $\begin{array}{c}\text { Experimento } 3 \\
\left(\mathrm{~g} .100 \mathrm{~g}^{-1}\right)\end{array}$ \\
\hline Farinha de trigo & 100 & 100 & 100 \\
\hline $\begin{array}{l}\text { Farinha de } \\
\text { algaroba }\end{array}$ & 25 & 5 & 15 \\
\hline $\begin{array}{l}\text { Gordura vegetal } \\
\text { de palma }\end{array}$ & 45,0 & 45,0 & 35,0 \\
\hline $\begin{array}{l}\text { Açúcar refinado } \\
\text { comum }\end{array}$ & 30,0 & 50,0 & 40,0 \\
\hline Lecitina de soja & 0,5 & 0,5 & 0,5 \\
\hline $\begin{array}{l}\text { Aroma de } \\
\text { baunilha }\end{array}$ & 0,5 & 0,5 & 0,5 \\
\hline Aveia & 5,0 & 5,0 & 5,0 \\
\hline Sal comum & 1,0 & 1,0 & 1,0 \\
\hline $\begin{array}{l}\text { Bicarbonato de } \\
\text { sódio }\end{array}$ & 1,5 & 1,5 & 1,5 \\
\hline $\begin{array}{l}\text { Bicarbonato de } \\
\text { amônia }\end{array}$ & 2,0 & 2,0 & 2,0 \\
\hline Amido de milho & 14,0 & 14,0 & 14,0 \\
\hline $\begin{array}{l}\text { Pirofosfato de } \\
\text { sódio }\end{array}$ & 0,8 & 0,8 & 0,8 \\
\hline
\end{tabular}

(porcentagem base de farinha). 
Caracterização tecnológica de cookies produzidos com diferentes concentrações de farinha de algaroba durante armazenamento por 120 dias

Gusmão, R. P. et al.

açúcar refinado comum (ALEGRE, Mamanguape-PB), amido de milho (MAIZENA, Igarassu-PE), bicarbonato de sódio (KITANO, São Bernardo do Campo-SP), bicarbonato de amônia (PANTEC, São Paulo-SP), pirofosfato de sódio (PLURY QUÍMICA, São Paulo-SP), gordura vegetal de palma (AGROPALMA, Belém-PA), lecitina de soja (PLURY QUÍMICA, São Paulo-SP), aveia (NESTLÉ, São Paulo-SP) e água filtrada. As porcentagens dos ingredientes foram calculadas tendo-se, como base, a quantidade de farinha de trigo (100\%).

\subsection{Produção dos biscoitos}

A mistura de ingredientes foi realizada em duas fases, na batedeira planetária, modelo BP-18, fabricante Prática Technipan; o tempo de mistura total foi de 10 minutos. A massa foi laminada horizontalmente com cilindro laminador, fabricante Perfecta, até espessura de $0,5 \pm 0,05 \mathrm{~cm}$. A massa foi formatada por uma matriz, que definiu as dimensões de $5 \mathrm{~mm}$ de espessura e $34 \mathrm{~mm}$ de diâmetro, e o formato circular do biscoito, por meio de corte. O forneamento foi realizado em forno industrial, fabricante Perfecta, modelo Millenium; nesta etapa, foi utilizada temperatura de $200^{\circ} \mathrm{C} \pm 5^{\circ} \mathrm{C}$ na zona central do forno. Após a estabilização da temperatura do forno, os biscoitos foram dispostos em esteira metálica no interior do forno, pelo tempo de 20 minutos. O resfriamento dos biscoitos foi realizado à temperatura ambiente, em bandejas de aço inox, por aproximadamente duas horas.

\subsection{Monitoramento dos parâmetros de qualidade durante o armazenamento}

Após a produção dos biscoitos, as amostras foram embaladas com embalagem primária de polipropileno biorientado metalizado (BOPP) e embalagem secundária de polietileno, sendo mantidas em sala em temperatura ambiente de $25^{\circ} \mathrm{C} \pm 3,0^{\circ} \mathrm{C}$. Os parâmetros de qualidade desses produtos foram acompanhados pelas análises de atividade de água, firmeza, fraturabilidade e cor. Para atividade de água, o biscoito foi macerado e cerca de $1 \mathrm{~g}$ foi inserido em cápsulas de polietileno, sendo então colocado no equipamento para medida direta da atividade de água, com tempo de análise de aproximadamente 20 minutos, para cada amostra. A análise de atividade de água foi realizada em equipamento Aqualab, fabricante Decagon Devices, modelo 3TE, Pullman, EUA, na temperatura constante de $25,0 \pm 0,3{ }^{\circ} \mathrm{C}$. Esta metodologia tem, por princípio, a medida do ponto de orvalho da amostra. A análise foi realizada em triplicata, conforme metodologia (BRASIL, 2005).

A análise da firmeza e da fraturabilidade dos biscoitos foi realizada 24 horas após o processamento dos biscoitos. A firmeza e a fraturabilidade instrumental foram avaliadas em texturômetro, modelo TA-XT2 (Stable Micro Systems, Surrey, Reino Unido). O probe utilizado foi o HDP/3PB (three point bending Rig), plataforma HDP/90. Os parâmetros utilizados nos testes foram: velocidade pré-teste $=1,0 \mathrm{~mm} / \mathrm{s}$, velocidade de teste $=3,0 \mathrm{~mm} / \mathrm{s}$, velocidade pós-teste $=10,0 \mathrm{~mm} / \mathrm{s}$, distância de 4,0 mm, com medida de força em compressão. Os resultados de firmeza e fraturabilidade (produto da gomosidade e elasticidade) foram expressos em newtons (N) e milímetros $(\mathrm{mm})$, respectivamente, e representaram a média aritmética de 10 determinações para amostras provenientes de um mesmo experimento.

A análise de cor foi realizada utilizando-se colorímetro Mini Scan Hunter Lab XE Plus, Reston, VA, EUA, sendo determinada de acordo com Sistema CIE-L*a* $b^{*}$ (Commision Internationale L'Eclairage), em que foram obtidos os parâmetros luminosidade $\left(L^{*}\right)$, intensidade de vermelho $\left(+a^{*}\right)$ e intensidade de amarelo $\left(+b^{*}\right)$, conforme metodologia de Altamirano-Fortoul e Rosell (2011). Esta análise foi realizada em decaplicata $(n=10)$.

O monitoramento dos parâmetros de qualidade foi realizado durante 120 dias de armazenamento, sendo avaliados periodicamente, com intervalos entre as medições de 15 dias.

\subsection{Caracterização física dos biscoitos}

A espessura e o diâmetro dos biscoitos enriquecidos com farinha de algaroba foram determinados antes e depois do forneamento, com o paquímetro digital, fabricante Panambra. O fator de expansão foi determinado pelo quociente entre o diâmetro médio e a espessura dos biscoitos, conforme descrito por Sharma et al. (2013). $\mathrm{O}$ volume específico dos biscoitos foi determinado pelo método 10-05.01 da AACC (2000), no mesmo dia em que foram processados. Estas determinações foram realizadas em decaplicata $(n=10)$

\subsection{Caracterização físico-química e determinação dos minerais}

As determinações de teor de água, cinzas, proteínas, lipídios e fibra bruta foram realizadas segundo metodologias descritas por (AACC, 2000), 24 horas após a produção dos biscoitos. As análises foram realizadas em triplicata.

Os minerais foram quantificados por fluorescência de raios $X$ por energia dispersiva, a partir das cinzas dos biscoitos. O espectrômetro de fluorescência de raios $X$ utilizado foi o modelo EDX-720, fabricante Shimadzu.

\subsection{Tratamento estatístico dos dados}

Foi utilizado um delineamento experimental fatorial, com dois fatores: nove períodos de armazenamento $(0,15,30,45,60,75,90,105$ e 120 dias) e três formulações de biscoitos formulados (experimentos 1, 2 e 3), para analisar a atividade de água, a firmeza e a fraturabilidade, 
Caracterização tecnológica de cookies produzidos com diferentes concentrações de farinha de algaroba durante armazenamento por $\mathbf{1 2 0}$ dias

Gusmão, R. P. et al.

e os parâmetros de cor: luminosidade $\left(L^{*}\right)$, intensidade de vermelho $\left(+a^{*}\right)$ e intensidade de amarelo $\left(+b^{*}\right)$.

Os resultados das análises físicas e físico-químicas, e da determinação dos minerais foram avaliados por meio do desvio padrão e análise de variância entre as repetições. Foi aplicado o Teste de Tukey, em nível de $5 \%$ de probabilidade. O software Assistat, versão 7.7 foi utilizado para todas as análises estatísticas.

\section{Resultados e discussão}

\subsection{Monitoramento dos parâmetros de qualidade}

Na Tabela 2 e na Figura 1, se encontram os comportamentos das variáveis atividade de água, firmeza, fraturabilidade, luminosidade $\left(L^{*}\right)$, intensidade de vermelho $\left(+a^{*}\right)$ e intensidade de amarelo $\left(+b^{*}\right)$ das três formulações de biscoitos, formulados com $25 \%$ de farinha de algaroba (experimento 1), $5 \%$ de farinha de

Tabela 2. Comportamento das variáveis atividade de água, firmeza, fraturabilidade e luminosidade durante 120 dias de armazenamento.

\begin{tabular}{|c|c|c|c|c|c|c|c|c|c|c|c|c|}
\hline \multirow{2}{*}{$\begin{array}{c}\text { Armazenamento } \\
\text { (dias) }\end{array}$} & \multicolumn{3}{|c|}{ Atividade de água } & \multicolumn{3}{|c|}{$\begin{array}{l}\text { Firmeza } \\
\text { (N) }\end{array}$} & \multicolumn{3}{|c|}{$\begin{array}{l}\text { Fraturabilidade } \\
\qquad(\mathrm{mm})\end{array}$} & \multicolumn{3}{|c|}{$\begin{array}{l}\text { Luminosidade } \\
\qquad\left(\mathrm{L}^{*}\right)\end{array}$} \\
\hline & Exp.1 & Exp.2 & Exp.3 & Exp.1 & Exp.2 & Exp.3 & Exp.1 & Exp.2 & Exp.3 & Exp.1 & Exp.2 & Exp.3 \\
\hline 0 & $0,263 \mathrm{fB}$ & $0,264 \mathrm{gB}$ &, $271 \mathrm{gA}$ & $25,89 a \mathrm{a}$ & $3,74 \mathrm{aC}$ & $26,65 a A$ & $0,41 \mathrm{cA}$ & $0,41 d$ & $0,42 c A$ & $46,2 \mathrm{gC}$ & 60 & $62,2 \mathrm{eA}$ \\
\hline 15 & $264 f B$ & $0,271 f A$ & $0,274 f g A$ & $23,51 \mathrm{bB}$ & $23,68 \mathrm{aB}$ & 25 & $0,42 c A$ & $0,42 \mathrm{~cd} A$ & $0,42 c A$ & $3 f C$ & eB & $65,8 \mathrm{~d} A$ \\
\hline 30 & $278 \mathrm{eA}$ & A & $0,279 f A$ & 23,0 & $a b B$ & 24 & $\mathrm{CB}$ &, $44 c A$ & $c A$ & $\mathrm{eC}$ & & $66,1 \mathrm{~d} A$ \\
\hline 45 & & $B$ & & 2 & $B$ & & $A$ & & 0,46 & & & \\
\hline 60 & $303 c B$ & $0,289 d C$ & $0,317 d A$ & $20,77 \mathrm{cC}$ & 2 & & 0,5 &, $48 b B$ & $B$ & $49,8 d C$ & & $67,8 \mathrm{cA}$ \\
\hline 75 & 332bA & $312 c C$ & $0,322 \mathrm{cB}$ & 20,4 & $A$ & & 0,5 & $0,48 b B$ & & $50,2 c C$ & $B$ & $68,2 \mathrm{bA}$ \\
\hline 90 & $0,345 a A$ & $0,332 \mathrm{bB}$ & $0,347 \mathrm{bA}$ & $19,68 \mathrm{deB}$ & $21,36 \mathrm{dA}$ & $21,89 d A$ & $0,52 \mathrm{aA}$ & $0,51 \mathrm{aA}$ & $0,52 a A$ & $50,2 c C$ & $66,5 \mathrm{bcB}$ & $68,2 \mathrm{bA}$ \\
\hline 105 & $0,345 \mathrm{aB}$ & $0,342 a B$ & $0,353 a A$ & $19,52 \mathrm{eB}$ & $21,27 d A$ & $20,74 \mathrm{eA}$ & $0,52 \mathrm{aA}$ & $0,52 \mathrm{aA}$ & $0,52 a A$ & $50,8 b C$ & $66,8 \mathrm{bB}$ & $68,4 \mathrm{bA}$ \\
\hline 120 & $0,347 a B$ & $0,342 \mathrm{aC}$ & $0,356 \mathrm{aA}$ & $19,38 \mathrm{eAB}$ & $18,76 \mathrm{eB}$ & $19,42 f \mathrm{~A}$ & $0,53 a A$ & $0,52 \mathrm{aA}$ & $0,52 \mathrm{aA}$ & $51,7 \mathrm{aC}$ & $67,9 a \mathrm{~B}$ & $71,1 \mathrm{aA}$ \\
\hline
\end{tabular}

Médias seguidas da mesma letra minúscula nas colunas e maiúscula nas linhas não diferem estatisticamente pelo Teste de Tukey a $5 \%$ de probabilidade. Exp. 1 - biscoito elaborado com $25 \%$ de farinha de algaroba; Exp. 2 - biscoito elaborado com $5 \%$ de farinha de algaroba; Exp.3 - biscoito elaborado com $15 \%$ de farinha de algaroba
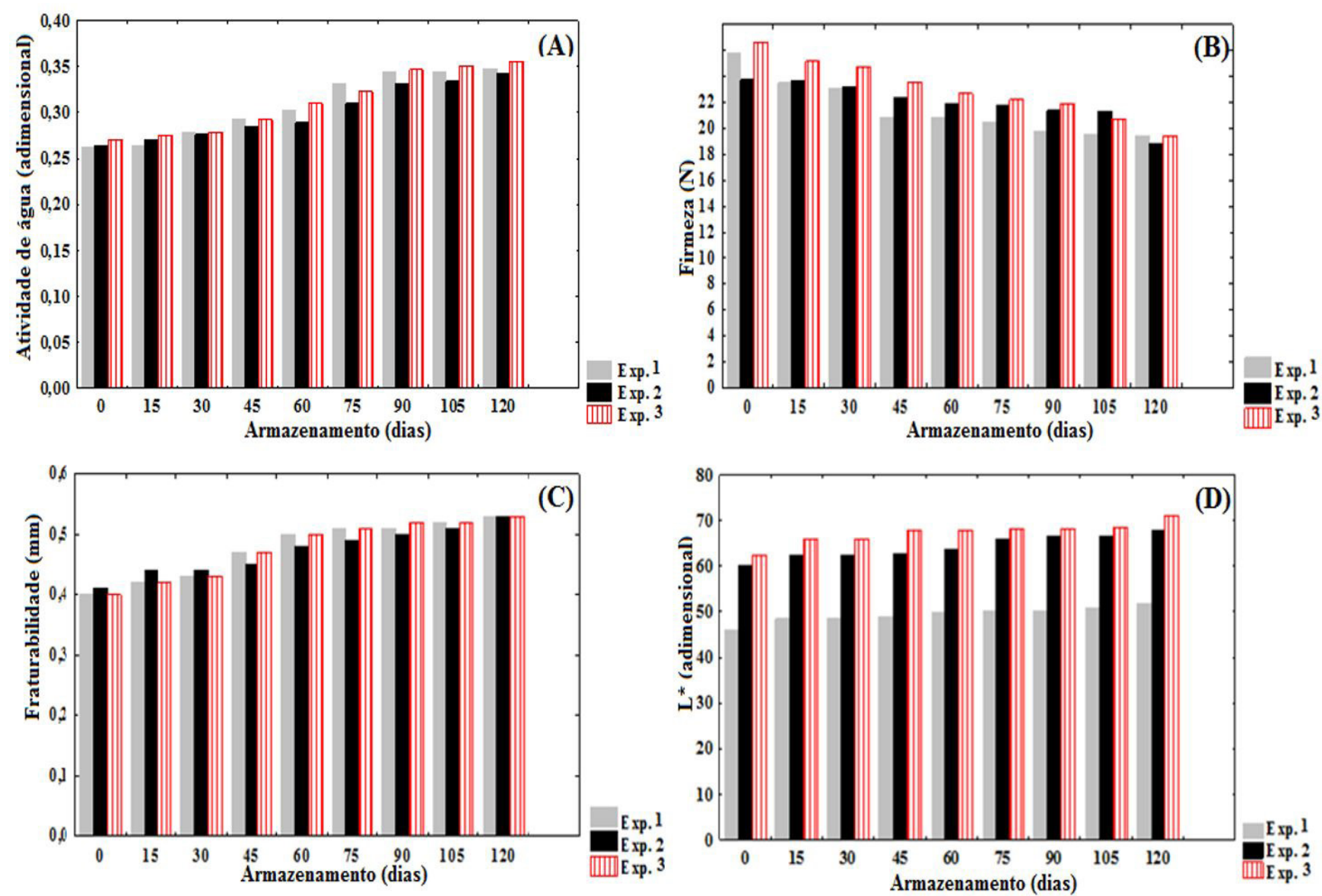

Figura 1. Comportamento das variáveis atividade de água, firmeza, fraturabilidade e luminosidade durante 120 dias de armazenamento. 
algaroba (experimento 2) e 15\% de farinha de algaroba (experimento 3), durante o armazenamento por 120 dias.

Analisando-se os resultados da Tabela 2 e da Figura 1 , percebe-se que houve incremento no valor da variável atividade de água, para todos os experimentos. Os valores de atividade de água, no início do armazenamento, eram de 0,263 a 0,271, e após 120 dias de armazenamento, estes foram de 0,342 a 0,356, para os biscoitos formulados com 25\% de farinha de algaroba, 30\% de teor de açúcar e $45 \%$ de teor de gordura de palma (experimento 1), $5 \%$ de farinha de algaroba, 50\% de teor de açúcar e $45 \%$ de teor de gordura de palma (experimento 2) e 15\% de farinha de algaroba, $40 \%$ de teor de açúcar e 35\% de teor de gordura de palma (experimento 3); contudo, esses valores estão dentro do limite considerado microbiologicamente estável, pois, de acordo com Cervenka et al. (2006), a atividade de água de biscoitos deve ser inferior a 0,6. $A$ atividade de água traz informações importantes sobre a vida útil de um produto, bem como é um parâmetro do produto a ser modificado durante o processo de produção, com o objetivo de aumentar a estabilidade dos alimentos. De acordo com Jardim (2010), mudanças físicas, como a crocância de biscoitos, dependem significativamente deste parâmetro.

Observa-se, ainda, na Tabela 2, que, a partir do período de 15 dias de armazenamento, o aumento da atividade de água cresce significativamente até 90 dias de armazenamento, para o biscoito formulado com $25 \%$ de farinha de algaroba (experimento 1). Para o biscoito formulado com 5\% de farinha de algaroba (experimento 2), a atividade de água aumenta significativamente do período inicial de armazenamento até 45 dias; a partir deste período até 60 dias, existe uma manutenção da atividade de água; após este período, a atividade de água aumenta significativamente até 105 dias de armazenamento. Já o quantitativo de atividade de água, para o biscoito formulado com 15\% de farinha de algaroba (experimento 3), teve um aumento significativo até 105 dias de armazenamento. Entretanto, a partir do período de 105 dias de armazenamento até os 120 dias, a atividade de água dos biscoitos permanece constante, para todos os experimentos.

Comparando-se as três formulações de biscoitos entre si, percebe-se que houve diferença estatística da atividade de água a 5\% de probabilidade, de acordo com o Teste de Tukey. De modo geral, o comportamento da atividade de água foi crescente para todas as formulações de biscoito e analisando-se, de maneira individual, as três formulações de biscoito ou as comparando entre si, percebe-se diferença de comportamento da atividade de água durante o armazenamento por 120 dias, o que pode ser explicado por variações nos parâmetros físicos dos biscoitos (espessura, massa, volume específico, diâmetro médio) e sobreposições aleatórias dos mesmos dentro das embalagens. Outro fator que deve ser levado em consideração é a diferença da atividade de água inicial dos biscoitos.

A variação do valor de atividade de água inicial e atividade de água final dos biscoitos, durante 120 dias de armazenamento, foi 31,93\%, 29,54\% e 31,37\%, para os experimentos 1, 2 e 3, respectivamente. Decorrido o período de 120 dias de armazenamento, este aumento de atividade de água pode ser justificado pela absorção de água do ambiente, devido à permeabilidade ao vapor de água da embalagem. Com o aumento da concentração de farinha de algaroba na formulação dos biscoitos, ocorreu maior variação entre a atividade de água inicial e final, durante o período de armazenamento, o que também pode ser explicado pela alta higroscopicidade da farinha de algaroba (GUSMÃO et al., 2016). Secchi et al. (2011) encontraram, estudando o armazenamento de biscoitos enriquecidos com farinha de amaranto, por 120 dias, variação maior que 30\% entre a atividade de água inicial e final.

Analisando-se a Tabela 2 e a Figura 1, percebe-se que houve alterações significativas na firmeza dos biscoitos em um nível de 5\% de probabilidade, pelo Teste de Tukey; para os experimentos 1, 2 e 3, a firmeza dos biscoitos diminuiu de $25,89 \mathrm{~N}$ para $19,38 \mathrm{~N}$, de $23,74 \mathrm{~N}$ para $18,76 \mathrm{~N}$ e de $26,65 \mathrm{~N}$ para $19,42 \mathrm{~N}$, respectivamente.

Para o biscoito elaborado com $25 \%$ de farinha de algaroba, 30\% de teor de açúcar e 45\% de teor de gordura de palma (experimento 1), percebe-se a maior perda de firmeza entre o período inicial de armazenamento (tempo 0) e 15 dias de armazenamento. A partir deste período, a firmeza vai sendo reduzida de forma significativa, a cada período de 30 dias de armazenamento, até o período de 90 dias de armazenamento; percebe-se uma manutenção da firmeza entre 90 dias de armazenamento até o período final de armazenamento (120 dias).

Com relação aos biscoitos elaborados a partir do experimento 2 ( $5 \%$ de farinha de algaroba), percebe-se uma manutenção do seu quantitativo de firmeza até o período de armazenamento de 30 dias; a partir deste período, nota-se uma redução da firmeza de forma significativa até o período de 120 dias de armazenamento. Já o biscoito elaborado com 15\% de farinha de algaroba, $40 \%$ de teor de açúcar e 35\% de teor de gordura de palma (experimento 3) não apresenta tendência definida quanto à redução da firmeza.

Ainda analisando-se a Tabela 2 e comparando-se as três formulações de biscoitos entre si, verifica-se que os valores médios de firmeza foram estatisticamente diferentes na maior parte do período de armazenamento, com comportamento decrescente durante 120 dias de armazenamento. Deve-se levar em consideração a diferença inicial de firmeza entre as três formulações de biscoitos, a qual pode ser atribuída à diferença de quantitativo de 
teor de farinha de algaroba, ao teor de gordura de palma e ao teor de açúcar nas formulações.

A variação da firmeza inicial e firmeza final dos biscoitos, durante o armazenamento por 120 dias, foi de $25,14 \%$ para o experimento $1,20,98 \%$ para o experimento 2 e $27,13 \%$ para o experimento 3 . De maneira geral, com o aumento do período de armazenamento, a firmeza dos biscoitos foi diminuindo para as três formulações de biscoito, o que pode ser explicado pelo aumento da atividade de água. As mudanças da firmeza dos biscoitos encontradas durante o armazenamento por 120 dias podem ser explicadas pela variação do teor de gordura nas formulações dos biscoitos (35 a 45\%). A gordura tem capacidade de reduzir o tempo de mistura e a energia exigidos, pois envolvem os grânulos de açúcar e as partículas de farinha de trigo. Esta também cumpre a função do controle e do desenvolvimento excessivo do glúten, tornando o produto final mais macio, com menor firmeza (BUENO, 2005).

A fraturabilidade é a tendência de um material a fratura, quebra ou desintegração, conforme sofre a aplicação de uma quantidade relativamente pequena de força ou impacto. Analisando-se os resultados da Tabela 2 e da Figura 1, percebe-se que a variável fraturabilidade apresentou diferença estatística durante o armazenamento por 120 dias, para os biscoitos elaborados a partir dos experimentos 1,2 e 3 . Ocorreu um aumento da fraturabilidade de $0,41 \mathrm{~mm}$ para $0,53 \mathrm{~mm}$, de $0,41 \mathrm{~mm}$ para $0,52 \mathrm{~mm}$ e de $0,42 \mathrm{~mm}$ para $0,52 \mathrm{~mm}$, para os biscoitos elaborados a partir dos experimentos 1,2 e 3 , respectivamente.

Ainda se percebe, analisando-se a Tabela 2, que o biscoito elaborado com $25 \%$ de farinha de algaroba, $30 \%$ de teor de açúcar e $45 \%$ de teor de gordura de palma (experimento 1) teve um aumento não significativo de fraturabilidade até 30 dias de armazenamento. Após este período, 0 aumento da fraturabilidade cresce significativamente até 60 dias de armazenamento, porém se nota, a partir deste período até os 120 dias de armazenamento, a manutenção da fraturabilidade dos biscoitos.

Para os biscoitos elaborados com $5 \%$ de farinha de algaroba (experimento 2), os valores da fraturabilidade foram estatisticamente diferentes até 90 dias de armazenamento e, decorrido este período até os 120 dias de armazenamento, houve um aumento não significativo.

O biscoito elaborado com $15 \%$ de farinha de algaroba, $40 \%$ de teor de açúcar e $35 \%$ de teor de gordura de palma (experimento 3) teve um aumento não significativo da fraturabilidade até 15 dias de armazenamento. Após este período, o aumento da fraturabilidade cresce significativamente até 75 dias de armazenamento; entretanto, a partir deste período até os 120 dias de armazenamento, nota-se a manutenção da fraturabilidade dos biscoitos.
Após a análise dos resultados da Tabela 2 e comparando-se as três formulações de biscoitos entre si, verifica-se que os valores médios de fraturabilidade foram estatisticamente iguais na maior parte do período de 120 dias de armazenamento. De modo geral, tal comportamento crescente da fraturabilidade dos biscoitos, durante o armazenamento por 120 dias, pode ser observado para as três formulações de biscoito.

A variação da fraturabilidade inicial e da fraturabilidade final, durante o armazenamento por 120 dias, foi de $29,27 \%, 26,83 \%$ e $23,81 \%$, para os experimentos 1,2 e 3 , respectivamente. Com o aumento do tempo de armazenamento, a fraturabilidade dos biscoitos foi aumentando para as três formulações de biscoitos, o que pode ser explicado pelo aumento da atividade de água dos biscoitos, ao longo do armazenamento por 120 dias. Conforme Reed et al. (2002) e Labuza et al. (2004), atividades de água entre 0,3 e 0,4, à temperatura ambiente, são suficientes para provocar alterações estruturais, como o empelotamento de pós e a perda de crocância e fraturabilidade em biscoitos. Ganokar \& Jain (2014), estudando o efeito da adição de farinha de linhaça em biscoitos, encontraram fraturabilidade variando de 0,54 a 0,65 mm.

Analisando-se os resultados da Tabela 2 e da Figura 1, percebe-se que a variável luminosidade $\left(L^{*}\right)$ apresentou diferença estatística significativa $(p<0,05)$, quando comparada às três formulações de biscoitos e as comparando entre si, durante 120 dias de armazenamento.

A variação da luminosidade ( $L^{*}$ ) foi de 46,2 para 51,7 , de 60,2 para 67,9 e de 62,2 para 71,1 , para os biscoitos formulados com $25 \%$ de farinha de algaroba, $30 \%$ de teor de açúcar e $45 \%$ de teor de gordura de palma (experimento 1), $5 \%$ de farinha de algaroba, $50 \%$ de teor de açúcar e $45 \%$ de teor de gordura de palma (experimento 2) e 15\% de farinha de algaroba, 40\% de teor de açúcar e $35 \%$ de teor de gordura de palma (experimento 3), respectivamente. Constata-se, em relação às três formulações, que as médias de luminosidade para os biscoitos elaborados com $25 \%$ de teor de farinha de algaroba (experimento 1) foram menores que as dos demais experimentos, para todos os períodos de armazenamento. Este resultado pode ser explicado pela existência de uma concentração maior da farinha de algaroba na formulação deste biscoito, quando comparada às demais formulações, já que a farinha de algaroba apresenta coloração mais escura que a coloração da farinha de trigo.

A variação da luminosidade inicial e a luminosidade final, durante 120 dias de armazenamento, foi de $11,90 \%$, $12,80 \%$ e $14,31 \%$, para os biscoitos elaborados a partir dos experimentos 1,2 e 3 , respectivamente; com o aumento do período de armazenamento, o parâmetro de luminosidade $\left(L^{*}\right)$ foi aumentando, ou seja, os biscoitos foram adquirindo coloração menos intensa. Devido à substituição da farinha de trigo por farinha de algaroba, existe uma redução do 
Caracterização tecnológica de cookies produzidos com diferentes concentrações de farinha de algaroba durante armazenamento por 120 dias

Gusmão, R. P. et al.

teor de glúten, ocorrendo desprendimento de algumas partículas de coloração mais clara do interior para a superfície dos biscoitos, o que justifica o aumento do parâmetro de luminosidade $\left(\mathrm{L}^{*}\right)$ (coloração mais clara). Este comportamento poderia ser reduzido com o aumento da quantidade de estabilizante lecitina de soja, na formulação dos biscoitos. Umesha et al. (2014) encontraram, estudando o armazenamento de biscoitos enriquecidos com ômega-3 microencapsulado, por 120 dias, variação do parâmetro de luminosidade $\left(L^{*}\right)$ de 61,1 a 69,2.

Constatou-se redução da intensidade de amarelo $\left(+b^{*}\right)$ e intensidade de vermelho $\left(+a^{*}\right)$, não apresentando tendência definida, em relação ao período de armazenamento por 120 dias, para os biscoitos elaborados com: 25\% de farinha de algaroba (experimento 1), $5 \%$ de farinha de algaroba (experimento 2) e 15\% de farinha de algaroba (experimento 3).

\subsection{Caracterização física dos biscoitos}

Na Tabela 3, estão os resultados das características físicas dos biscoitos antes do forneamento e depois deste.

Percebe-se, analisando-se a Tabela 3, que houve diferença significativa $(p<0,05)$, quando comparadas, entre si, as três formulações de biscoito antes do forneamento e depois do forneamento, em relação aos parâmetros físicos (espessura, diâmetro médio, fator de expansão, volume específico e massa). Os parâmetros massa, espessura, diâmetro médio e volume específico não tiveram diferença significativa quando são comparadas as três formulações entre si, para os biscoitos antes do forneamento e depois do forneamento.

A massa dos biscoitos antes de forneamento variou entre $7,32 \mathrm{~g}$ e 7,20 g, e depois do forneamento, entre $6,10 \mathrm{~g}$ e 6,20 g. Os biscoitos perderam, após o tempo de forneamento, aproximadamente $15 \%$ de sua massa, devido à redução do teor de água. Com relação à espessura, ocorreu um aumento de aproximadamente $75 \%$, com valores variando entre $0,52 \mathrm{~cm}$ e 0,92 cm, para os biscoitos antes do forneamento e depois do forneamento. O volume específico dos biscoitos aumentou mais de $100 \%$, variando entre $0,75 \mathrm{~cm}^{3} \cdot \mathrm{g}^{-1}$ e 1,62 $\mathrm{cm}^{3} \cdot \mathrm{g}^{-1}$, para os biscoitos elaborados com $25 \%$ de farinha de algaroba (experimento 1 antes do forneamento) e 15\% de farinha de algaroba (experimento 2 após o forneamento). O aumento desses parâmetros pode ser justificado pela adição de fermento químico (pirofosfato ácido de sódio) na formulação dos biscoitos.

Como se observa na Tabela 4, os biscoitos tiveram seus fatores de expansão variando entre 6,43 e 6,67, e entre 3,80 e 3,83 , antes do forneamento e depois do forneamento, respectivamente. À medida que a concentração de farinha de algaroba aumenta nos biscoitos, de 5\% para o biscoito elaborado a partir do experimento 2, a 25\% para o biscoito elaborado a partir do experimento 1, o fator de expansão foi reduzido; esta redução pode ser explicada pelo acréscimo de farinha de algaroba na formulação dos biscoitos, já que a matéria-prima é rica em fibras e possui maior capacidade de absorção de água, limitando o fator de expansão. Os efeitos negativos ocasionados na expansão dos biscoitos pela suplementação com farinhas ricas em fibras já foram verificados, em maior ou menor grau, por diversos pesquisadores. De modo geral, a expansão dos biscoitos diminui sempre que o nível de substituição de farinha de trigo aumenta (CHUNG et al., 2014; ZUCCO et al., 2011).

\subsection{Caracterização físico-química e determinação dos minerais}

Os resultados da caracterização físico-química e da determinação dos minerais dos biscoitos estão apresentados na Tabela 4.

Analisando-se a Tabela 4, percebe-se que houve diferença significativa $(p<0,05)$ nos teores de cinzas,

Tabela 3. Massa $(\mathrm{g})$, diâmetro médio $(\mathrm{cm})$, espessura média $(\mathrm{cm})$, fator de expansão e volume específico $\left(\mathrm{cm}^{3} \cdot \mathrm{g}^{-1}\right)$ dos biscoitos antes e depois do forneamento.

\begin{tabular}{|c|c|c|c|c|c|}
\hline Amostra & $\begin{array}{l}\text { Espessura } \\
\text { média } \\
\text { (cm) }\end{array}$ & $\begin{array}{l}\text { Diâmetro médio } \\
\text { (cm) }\end{array}$ & $\begin{array}{l}\text { Fator de } \\
\text { expansão }\end{array}$ & $\begin{array}{l}\text { Volume específico } \\
\qquad\left(\mathrm{cm}^{3} \cdot \mathrm{g}^{-1}\right)\end{array}$ & $\begin{array}{c}\text { Massa } \\
\text { (g) }\end{array}$ \\
\hline $1 \mathrm{AF}$ & $0,53 \pm 0,01^{b}$ & $3,41 \pm 0,17^{b}$ & $6,43 \pm 0,03^{c}$ & $0,75 \pm 0,02^{b}$ & $7,23 \pm 0,10^{a}$ \\
\hline $2 \mathrm{AF}$ & $0,51 \pm 0,02^{b}$ & $3,40 \pm 0,24^{b}$ & $6,67 \pm 0,15^{a}$ & $0,75 \pm 0,03^{b}$ & $7,32 \pm 0,15^{a}$ \\
\hline $3 \mathrm{AF}$ & $0,52 \pm 0,02^{b}$ & $3,41 \pm 0,25^{b}$ & $6,56 \pm 0,50^{b}$ & $0,76 \pm 0,03^{b}$ & $7,20 \pm 0,14^{a}$ \\
\hline DMS & 0,0456 & 0,6446 & 1,0094 & 0,0667 & 0,2400 \\
\hline $1 \mathrm{DF}$ & $0,92 \pm 0,02^{a}$ & $3,50 \pm 0,06^{a}$ & $3,80 \pm 0,02^{e}$ & $1,61 \pm 0,10^{a}$ & $6,20 \pm 0,15^{b}$ \\
\hline $2 \mathrm{DF}$ & $0,91 \pm 0,03^{a}$ & $3,52 \pm 0,10^{a}$ & $3,87 \pm 0,10^{d}$ & $1,62 \pm 0,05^{a}$ & $6,11 \pm 0,05^{b}$ \\
\hline $3 \mathrm{DF}$ & $0,92 \pm 0,02^{\mathrm{a}}$ & $3,52 \pm 0,15^{a}$ & $3,83 \pm 0,07 e$ & $1,62 \pm 0,07^{a}$ & $6,10 \pm 0,10^{b}$ \\
\hline DMS & 0,0855 & 0,2729 & 0,1889 & 0,1467 & 0,3335 \\
\hline
\end{tabular}

Em uma mesma coluna, médias com letras em comum não diferem significativamente ( $\mathrm{p}<0,05)$. DMS - desvio mínimo significativo; Exp. 1 - biscoito elaborado com $25 \%$ de farinha de algaroba; Exp. 2 - biscoito elaborado com $5 \%$ de farinha de algaroba; Exp. 3 - biscoito elaborado com $15 \%$ de farinha de algaroba; AF - antes do forneamento; DF - depois do forneamento. 
Caracterização tecnológica de cookies produzidos com diferentes concentrações de farinha de algaroba durante armazenamento por 120 dias

Gusmão, R. P. et al.

Tabela 4. Caracterização físico-química e determinação dos minerais dos biscoitos enriquecidos com farinha de algaroba.

\begin{tabular}{|c|c|c|c|}
\hline \multirow{2}{*}{ Determinação } & \multicolumn{3}{|c|}{ Valor médio \pm desvio padrão } \\
\hline & Exp. 1 & Exp. 2 & Exp. 3 \\
\hline Teor de água $\left(\mathrm{g} .100 \mathrm{~g}^{-1}\right)$ & $4,42 \pm 0,01^{a}$ & $4,12 \pm 0,01^{b}$ & $4,37 \pm 0,02^{a}$ \\
\hline Cinzas $\left(\mathrm{g} .100 \mathrm{~g}^{-1}\right)$ & $1,52 \pm 0,02^{a}$ & $1,40 \pm 0,03^{c}$ & $1,48 \pm 0,01^{b}$ \\
\hline Proteínas $\left(\mathrm{g} .100 \mathrm{~g}^{-1}\right)$ & $7,40 \pm 0,10^{a}$ & $7,11 \pm 0,12^{\mathrm{c}}$ & $7,20 \pm 0,13^{b}$ \\
\hline Lipídios $\left(\mathrm{g} .100 \mathrm{~g}^{-1}\right)$ & $29,92 \pm 0,15^{b}$ & $31,05 \pm 0,17^{a}$ & $28,06 \pm 0,21^{\circ}$ \\
\hline Carboidratos $\left(\mathrm{g} .100 \mathrm{~g}^{-1}\right)$ & $47,93 \pm 0,11^{c}$ & $54,68 \pm 0,09^{a}$ & $51,20 \pm 0,13^{b}$ \\
\hline Fibras $\left(\mathrm{g} .100 \mathrm{~g}^{-1}\right)$ & $2,53 \pm 0,15^{a}$ & $2,48 \pm 0,12^{\mathrm{a}}$ & $2,50 \pm 0,13^{b}$ \\
\hline Açúcares totais $\left(\mathrm{g} .100 \mathrm{~g}^{-1}\right)$ & $45,40 \pm 0,14^{c}$ & $52,20 \pm 0,09 a$ & $48,70 \pm 0,10^{b}$ \\
\hline Cálcio (mg.100g-1) & $430,94 \pm 0,23^{a}$ & $415,57 \pm 0,20^{\circ}$ & $421,19 \pm 0,19^{b}$ \\
\hline Fósforo (mg.100g-1) & $587,48 \pm 0,30^{a}$ & $554,48 \pm 0,24^{c}$ & $574,32 \pm 0,25^{b}$ \\
\hline Ferro (mg. $\left.100 \mathrm{~g}^{-1}\right)$ & $23,16 \pm 0,23^{a}$ & $20,05 \pm 0,31^{c}$ & $22,33 \pm 0,19^{b}$ \\
\hline
\end{tabular}

Em uma mesma linha, médias com letras em comum não diferem significativamente ( $<<0,05)$. Exp.1 - biscoito elaborado com $25 \%$ de farinha de algaroba; Exp. 2 - biscoito elaborado com 5\% de farinha de algaroba; Exp.3 - biscoito elaborado com 15\% de farinha de algaroba.

proteínas, lipídios, fibra bruta, cálcio, fósforo e ferro, entre as três formulações de biscoitos enriquecidos com farinha de algaroba; com o aumento da concentração de algaroba, ocorreu aumento dos teores de cinzas, fibras, cálcio, fósforo e ferro nos biscoitos. O quantitativo de lipídios não foi crescente com o aumento da concentração de farinha de algaroba, em virtude da maior concentração de teor de gordura de palma presente na formulação dos biscoitos elaborados com 25\% de farinha de algaroba, $30 \%$ de teor de açúcar, $45 \%$ de teor de gordura de palma (experimento 1) e 5\% de farinha de algaroba, 50\% de teor de açúcar, $45 \%$ de teor de gordura de palma (experimento 2).

O teor de água dos biscoitos enriquecidos com farinha de algaroba variou de 4,12 g. $100 \mathrm{~g}^{-1}$ a 4,42 g. $100 \mathrm{~g}^{-1}$. Inglett et al. (2015) encontraram valores de teor de água variando de 3,16 g. $100 \mathrm{~g}^{-1}$ a 3,95 g. $100 \mathrm{~g}^{-1}$, estudando as propriedades físicas de biscoitos sem glúten, produzidos com farinha de amaranto e aveia. Com referência ao teor de cinzas, foram encontrados valores variando de $1,40 \mathrm{~g} .100 \mathrm{~g}^{-1}$ a $1,52 \mathrm{~g} .100 \mathrm{~g}^{-1}$, para os biscoitos elaborados com $5 \%$ de farinha de algaroba (experimento 2) e $25 \%$ de farinha de algaroba (experimento 3). Bassinello et al. (2011) encontraram teor de cinzas variando de $0,82 \mathrm{~g} \cdot 100 \mathrm{~g}^{-1}$ a $2,2 \mathrm{~g} \cdot 100 \mathrm{~g}^{-1}$, com diferentes concentrações de farinha de arroz (0, 20, 40 e 60\%), na formulação de biscoitos, sendo que o teor de proteína dos biscoitos variou de $7,11 \mathrm{~g} .100 \mathrm{~g}^{-1}$ a $7,40 \mathrm{~g} .100 \mathrm{~g}^{-1}$. Ertas (2015) encontrou, estudando a caracterização de biscoitos elaborados com diferentes níveis de farelo de trigo estabilizado (10, 20 e 30\%), teores de proteína variando de 18,06 g.100 g-1 a 19,30 g. $100 \mathrm{~g}^{-1}$. Em relação ao teor de lipídios, os valores encontrados apresentaram variação de $28,06 \mathrm{~g} \cdot 100 \mathrm{~g}^{-1} \mathrm{a}$

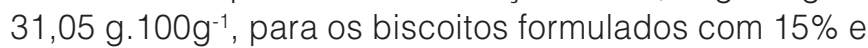
$5 \%$ de farinha de algaroba, respectivamente. Park et al. (2015) verificaram, estudando o desenvolvimento de biscoito elaborado com diferentes frações de farinha de soja e okara, valores de lipídios próximos ao deste estudo, variando

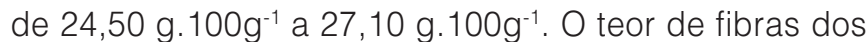
biscoitos variou de 2,48 g. $100 \mathrm{~g}^{-1}$ a 2,53 g. $100 \mathrm{~g}^{-1}$, valores inferiores a 4,63 g. $100 \mathrm{~g}^{-1}$ e 5,83 g. $100 \mathrm{~g}^{-1}$, encontrados por Duta e Culetu (2015), estudando biscoitos isentos de glúten produzidos com farinha e farelo de aveia.

Com relação aos minerais, foram encontrados os valores de cálcio, fósforo e ferro variando de $415,57 \mathrm{mg} .100 \mathrm{~g}^{-1}$ a $650,75 \mathrm{mg} .100 \mathrm{~g}^{-1}$, de $554,48 \mathrm{mg} .100 \mathrm{~g}^{-1}$ a $587,78 \mathrm{mg} .100 \mathrm{~g}^{-1}$ e de $20,05 \mathrm{mg} .100 \mathrm{~g}^{-1}$ a $23,16 \mathrm{mg} .100 \mathrm{~g}^{-1}$, respectivamente. Mesías et al. (2015) encontraram, na composição de biscoito, um teor de cálcio e ferro de

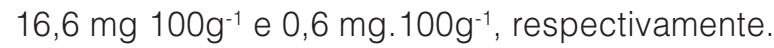

\section{Conclusão}

Os resultados da pesquisa mostraram que, decorrido o período de 120 dias de armazenamento, os biscoitos formulados com $25 \%$ de farinha de algaroba (experimento 1), $5 \%$ de farinha de algaroba (experimento 2) e 15\% de farinha de algaroba (experimento 3) tiveram seus valores de atividade de água, fraturabilidade e luminosidade aumentados e de firmeza reduzida, tendo como motivo principal a substituição da farinha de trigo, devido à alta higroscopicidade e ao baixo valor de luminosidade dessa matéria-prima. A variação do teor de farinha de algaroba na formulação dos biscoitos gerou diferenças físicas significativas entre os experimentos antes e depois do forneamento. Os biscoitos apresentaram elevada concentração de nutrientes (cálcio, ferro e fósforo) e comportamento similar ao de produtos elaborados com outras matérias-primas já existentes no mercado, durante o armazenamento.

\section{Referências}

ALTAMIRANO-FORTOUL, R.; ROSELL, C. M. Physico-chemical changes in breads from bake off technologies during storage. Food Science and Technology, v. 44, n. 3, p. 631-636, 2011.

AMERICAN ASSOCIATION OF CEREAL CHEMISTS - AACC. Approved methods of the American Association of Cereal Chemists. 10th ed. Saint Paul: AACC, 2000. 
Caracterização tecnológica de cookies produzidos com diferentes concentrações de farinha de algaroba durante armazenamento por 120 dias

Gusmão, R. P. et al.

BASSINELLO, P. Z.; FREITAS, D. G. C.; ASCHERI, J. L. R.; TAKEITI, C. Y.; CARVALHO, R. N.; KOAKUZU, S. N.; CARVALHO, A. V. Characterization of cookies formulated with rice and black bean extruded flours. Procedia Food Science, v. 1, p. 1645-1652, 2011. http://dx.doi.org/10.1016/j.profoo.2011.09.243.

BRASIL. Ministério da Saúde. Agência de Vigilância Sanitária. Resolução n²63, de 22 de setembro de 2005. Regulamento Técnico para produtos de cereais, amidos, farinhas e farelos. Diário Oficial [da] República Federativa do Brasil, Brasília, DF, 23 set. 2005.

BUENO, R. O. G. Características de qualidade de biscoitos e barras de cereais ricos em fibra alimentar a partir de farinha de semente e polpa de nêspera. 2005. 103 f. Dissertação (Mestrado em Tecnologia de Alimentos)--Universidade Federal do Paraná, Curitiba.

CERVENKA, L.; BROZKOVÁ, I.; VYTRASOVÁ, J. Effects of the principal ingredients of biscuits upon water activity. Journal of Food and Nutrition Research, v. 45, p. 39-43, 2006.

CHUNG, H.-J.; CHO, A.; LIM, S. T.; MORALES, F.-J. Utilization of germinated and heat-moisture treated brown rices in sugarsnap cookies. LWT. Food Science and Technology, v. 57, p. 260-266, 2014

DUTA, D. E.; CULETU, A. Evaluation of rheological, physicochemical, thermal, mechanical and sensory properties of oat-based gluten free cookies. Journal of Food Engineering, v. 162, p. 1-8, 2015. http://dx.doi.org/10.1016/j.jfoodeng.2015.04.002.

ERTAS, N. Effect of wheat bran stabilization methods on nutritional and physico-mechanical characteristics of cookies. Journal of Food Quality, v. 38, n. 3, p. 184-191, 2015. http://dx.doi. org/10.1111/jfq. 12130 .

GANORKAR, P. M.; JAIN, R. K. Effect of flaxseed incorporation on physical, sensorial, textural and chemical attributes of cookies. International Food Research Journal, Selangor, v. 21, n. 4, p. 1515-1521, 2014.

GUSMÃO, R. P.; CAVALCANTI-MATA, M. E. M. R.; DUARTE, M. E. M.; GUSMÃO, T. A. S. Particle size, morphological, rheological, physicochemical characterization and designation of minerals in mesquite flour (Proposis julifrora). Journal of Cereal Science, v. 69, p. 119-124, 2016. http://dx. doi.org/10.1016/j.jcs.2016.02.017.

INGLETT, G. E.; CHEN, D.; LIU, S. X. Physical properties of gluten-free sugar cookies made from amaranth-oat composites. LWT. Food Science and Technology, v. 63, p. 214-220, 2015.

JARDIM, D. C. P. Atividade de água e a estabilidade dos alimentos. In: MOURA, S. C. S. R.; GERMER, S. P. M. (Coord.). Reações de transformação e vida-de-prateleira de alimentos processados. 4. ed. Campinas: ITAL, 2010. p. 17-23. Manual Técnico, n. 6).

LABUZA, T.; ROE, K.; PAYNE, C.; PANDA, F.; LABUZA, T. J.; LABUZA, P. S.; KRUSCH, L. Storage stability of dry food systems: influence of state changes during drying and storage. In: INTERNATIONAL DRYING SYMPOSIUM - IDS'2004, 14.,
2004, São Paulo. Proceedings... São Paulo: Ourograf Gráfica e Editora, 2004. p. 48-68.

LEMOS, A. R.; CAPRILES, V. D.; SILVA, M. E. M. P.; ARÊAS, J. A. $G$. Effect of incorporation of amaranth on the physical properties and nutritional value of cheese bread. Ciência e Tecnologia de Alimentos, v. 32, n. 3, p. 427-431, 2012. http://dx.doi.org/10.1590/ S0101-20612012005000079.

MARTINEZ, C. S.; RIBOTTA, P. D.; AÑÓN, M. C.; LEÓN, A. E. Effect of amaranth flour (Amaranthus mantegazzianus) on the technological and sensory quality of bread wheat pasta. Food Science \& Technology International, v. 20, n. 2, p. 127-135, 2014. PMid:23733824. http://dx.doi.org/10.1177/1082013213476072.

MESÍAS, M.; HOLGADO, F.; MARQUEZ-RUIZ, G.; MORALES, F. $J$. Effect of sodium replacement in cookies on the formation of process contaminants and lipid oxidation. Food Science and Technology, v. 62, p. 633-639, 2015.

PARK, J.; CHOI, I.; KIM, Y. Cookies formulated from fresh okara using starch, soy flour and hydroxypropyl methylcellulose have high quality and nutricional value. Lebensmittel-Wissenschaft + Technologie, v. 63, n. 1, p. 660-666, 2015. http://dx.doi. org/10.1016/j.lwt.2015.03.110.

RAMOS, N. C.; PIEMOLINI-BARRETO, L. T.; SANDRI, I. G. Elaboração de pré-mistura para bolo sem glúten. Alimentos e Nutrição, v. 23, n. 1, p. 33-38, 2012.

REED, K. A.; SIMS, C. A.; GORBET, D. W.; ÓKEEFE, S. F. Storage water activity affects flavor fade in high and normal oleic peanuts. Food Research International, v. 35, n. 8, p. 769-774, 2002. http://dx.doi.org/10.1016/S0963-9969(02)00073-X.

ROBERTSON, R. L. Food packaging: principles and practice. 2nd ed. Boca Raton: Taylor \& Francis, 2006. 568 p.

SECCHI, N.; STARA, G.; ANEDDA, R.; CAMPUS, M.; PIGA, A.; ROGGIO, T.; CATZEDDU, P. Effectiveness of sweet ovine whey powder in increasing the shelf life of Amaretti cookies. Lebensmittel-Wissenschaft + Technologie, v. 44, n. 4, p. 1073-1078, 2011. http://dx.doi.org/10.1016/j.Iwt.2010.09.018.

SHARMA, P.; VELU, V.; INDRANI, D.; SINGH, R. P. Effect of dried guduchi (Tinosporacordifolia) leaf powder on rheological, organoleptic and nutritional characteristics of cookies. Food Research International, v. 50, n. 2, p. 704-709, 2013. http:// dx.doi.org/10.1016/j.foodres.2012.03.002.

UMESHA, S. S.; MANOHAR, R.; INDIRAMMA, A. R.; AKSHITHA, S.; AKHILENDER NAIDU, K. Enrichment of biscuits with microencapsulated omega-3 fatty acid (Alpha-linolenic acid) rich Garden cress (Lepidiumsativum) seed oil: physical, sensory and storage quality characteristics of biscuits. LebensmittelWissenschaft + Technologie, v. 1, p. 1-8, 2014.

ZUCCO, F.; BORSUK, Y.; ARNTFIELD, S. D. Physical and nutritional evaluation of wheat cookies supplemented with pulse flours of different particle sizes. Lebensmittel-Wissenschaft + Technologie, v. 44, n. 10, p. 2070-2076, 2011. http://dx.doi. org/10.1016/j.Iwt.2011.06.007. 